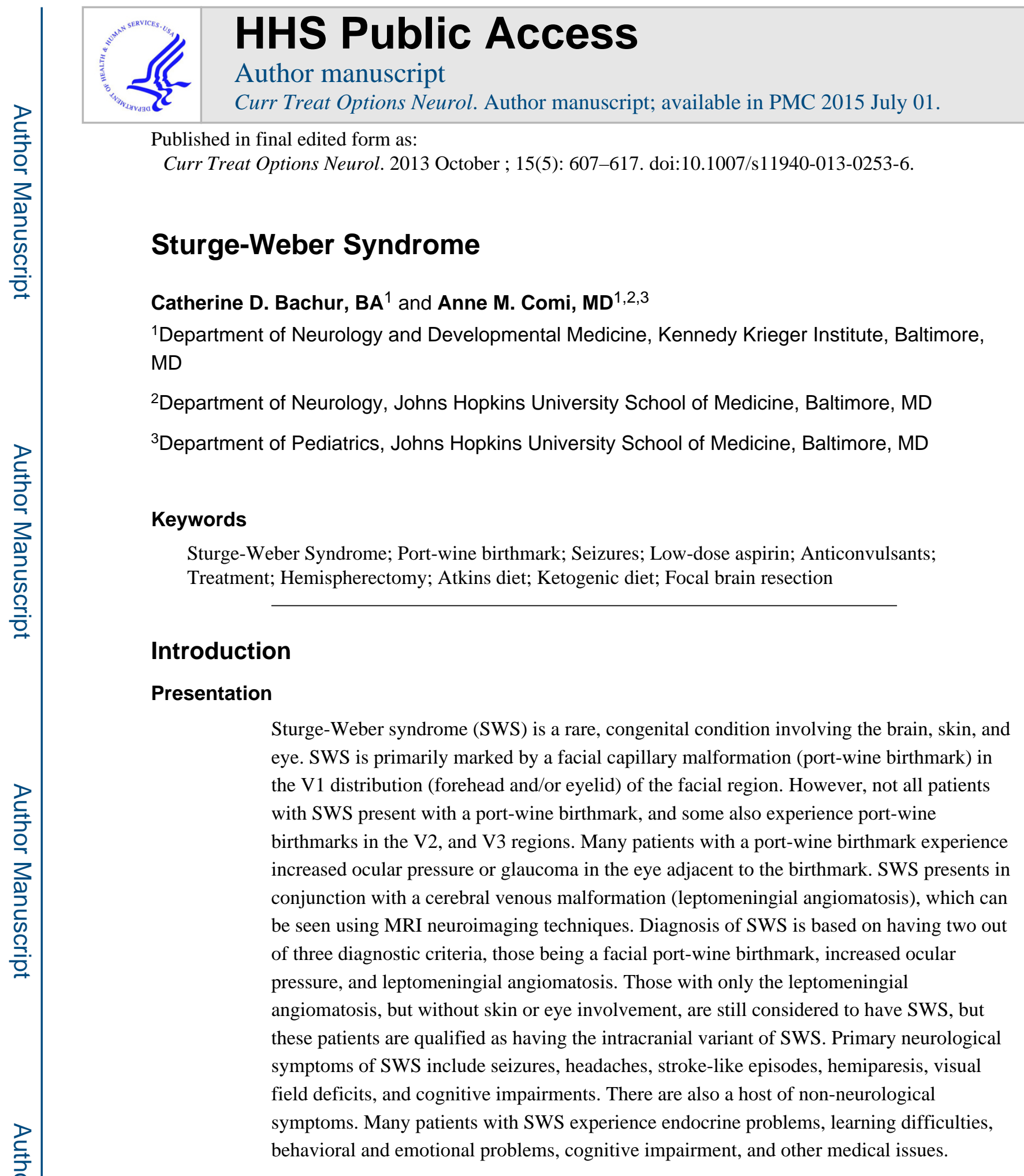

Corresponding author: Anne M. Comi, MD, Hunter Nelson Sturge-Weber Clinic, Kennedy Krieger Institute, 801 N. Broadway, Room 553, Baltimore, MD 21205, Phone: 443-923-9127, Fax: 443-923-9540, comi@kennedykrieger.org.

Compliance with Ethics Guidelines

Conflict of Interest

Anne M. Comi has two patents pending regarding the GNAQ gene mutation and potential treatment approaches based on this mechanism.

Catherine D. Bachur declares that she has no conflict of interest.

Human and Animal Rights and Informed Consent

This article does not contain any studies with human or animal subjects performed by any of the authors. 


\section{Diagnosis of SWS}

Only $8-20 \%$ of patients with facial port-wine birthmarks, with and without ocular involvement, develop neurological symptoms [3, 4]. Those with only V2 and/or V3 involvement have a significantly lower risk for experiencing symptoms of SWS, and early screening is less urgent The elevated risk really lies with the infants having a V1 distribution port-wine birthmark $[1,2,3]$. When extensive bilateral facial port-wine birthmark (involving both $\mathrm{V} 1$ distributions) is present, then the risk of brain involvement is somewhat higher (about 35\%) and the threshold for MRI imaging these infants is probably lower for most physicians [4]. While many children with port-wine birthmarks in the V1 region do not go on to develop other symptoms of SWS, early screening is essential in order to aggressively manage potential risks. The majority present with focal or complex partial seizures; less common presentations include early handedness or a visual gaze preference. Several imaging techniques are readily used to help diagnose SWS and to determine extent of brain involvement. Magnetic resonance imaging (MRI) is the generally accepted preference for diagnosing and monitoring brain involvement, particularly in patients over one year of age. The ideal standard is a T1 and T2-weighted brain MRI with gadolinium contrast, and postcontrast fluid attenuated inversion recovery (FLAIR) [ $\left.5^{*}\right]$. Neuroimaging can be done proactively, before symptoms even occur. However, in newborns and young infants, these imaging techniques can often lead to false negative results [6]. For this reason, MRI is not suggested for young infants as it can be falsely reassuring, and imaging all these children (most of whom are normal) would require the sedation of large numbers of normal children. However, it is highly recommended for high risk patients as soon as possible after turning one year of age or if symptoms begin in the meantime. However, it is highly recommended for high risk patients as soon as possible after turning one year of age. As mentioned before, early diagnosis of brain involvement is important to either reassure parents or provide a basis for prophylactic treatments. In younger patients, electroencephalography (EEG) is a good option for assessing abnormal brain activity, and identifying patients at risk for future neurologic symptoms [6]. While EEG can also lead to false negatives in young patients who may not have fully developed symptoms, EEG is a useful method because it is non-invasive and can be repeated easily, and safely as often as necessary. Diagnosis is never made by EEG; a concerning EEG must be followed by appropriate imaging; either at the time of a concerning EEG or later after a year of age. The value of EEG/quantitative EEG is in aiding the selection of the few infants who should undergo early MRI imaging. Quantified EEG results, which correlate with clinical SWS severity, can help to minimize variation in clinical interpretation of EEGs [7]. FDG-PET and MR spectroscopy can be used to help screen surgical candidates, or as research tools, but are not generally used as routine clinical tests for diagnosis or monitoring disease severity.

\section{Pathogenesis}

As recently reported, SWS is caused by a somatic mutation in a nucleotide transition in the gene GNAQ on chromosome 9q21. The mutation in GNAQ increases activity in pathways transmitting signals from a subset of G protein coupled receptors (GCPR). Mutant constructs were transiently expressed in T293 cells and demonstrated constitutive over activation of downstream pathways. Precisely how this over activation results in port-wine birthmarks and SWS requires additional study [8**]. Further research is being done to determine the 
cell type(s) containing this somatic mutation, additional pathways impacted, develop animal and in vitro models, and to create potential therapies for reversing the over activation. This discovery opens the door to a host of new research into SWS pathogenesis and treatment. At this point in time, genetic testing is not clinically available for SWS, nor does it have a role in separating an isolated port-wine birthmark from SWS. However, as research advances on the genetic basis of the disorder, testing may be useful in the future.

\section{Natural History}

Most patients with brain involvement will begin seizure activity during infancy, but new neurological symptoms have been shown to present in adulthood in certain cases $[9,10,11]$. Adult-onset SWS does not necessarily follow the same pattern as early onset SWS, but has not been studied extensively. Seizures in these patients tend to be easier to control and less associated with strokes and neurologic deterioration. Neurological symptoms can continue to worsen gradually with time, or are often caused by a specific stroke-like episode. No consensus exists on what a stroke-like episode is, but these episodes can be functionally defined as a new neurological deficit, occurring with or without the context of seizures, which last longer than 24 hours. These episodes may be triggered by falls, head trauma, headaches, or occur randomly $[9,12]$. Many of these neurologic deficits fully or partially resolve but in young children are more likely to result in accrual of permanent disability. Seizures occur in roughly $75 \%$ of patients with unilateral brain involvement, and $95 \%$ of patients with bilateral brain involvement $[13,14]$. It is essential to educate parents on recognizing seizures, as SWS seizures often do not present in the manner that parents expect. Often infantile seizures are subtle, rhythmic twitching of the hand, foot, or eye. Many people expect seizures to be generalized tonic-clonic activity, but this is not often seen in SWS [9]. If parents are not properly educated on the presentation of seizures, they may miss them altogether. If seizures continue without being controlled, they can drastically worsen cognitive impairments and neurological injury [15**, 16, 17]. Typically, neurologic status stabilizes by school age and this correlates with a stabilization in the glucose hypometabolism noted on PET imaging [18]; likely this is in part due to the increased seizure threshold in older children and in the opening of collateral deep draining vessels which improve venous outflow and therefore stabilize perfusion to that region. Migraines can begin at a very young age and may trigger seizures and stroke-like episodes. At the same time, seizures and stroke-like episodes can trigger migraines [19, 9]. In adolescence, stable epilepsy, stroke-like episodes, and migraines may become a greater issue once again although not usually with the same degree of deterioration as in infancy and toddler years. Generally neurological status is more stable in adults with SWS although older adults may present with new onset of neurologic deficits, memory or mood issues and worsening of epilepsy [9]. The neurological trajectory in older adults with SWS has not been well defined in older adults with SWS and is poorly understood.

\section{Treatment}

Seizures

The primary aim of pharmacologic treatment is to minimize, or optimally eliminate seizure activity. It is likely that lengthy and frequent seizures help to worsen neurological injury, so 
controlling these seizures in the long term, and treating them at the time of occurrence are both very important $[9,16,17]$. There are a variety of anti-convulsant medications, which may be successful in a variety of situations. We recommend starting an anticonvulsant after the first focal seizure and the most common first line anticonvulsant is oxcarbazepine [9]. However, this may occasionally lead to central hypothyroidism, especially in girls [20]. Other first line anticonvulsants to consider include levetiracetam and topiramate [9]. Other potential, but less commonly used chronic anticonvulsants, include valproic acid, carbamazepine, zonisamide, lamotrigine, and phenobarbital. Oftentimes, seizures are precipitated by outside factors such as stress, lack of sleep, and illness. While it is impossible to fully control these factors altogether, it is essential to try and minimize their negative influence. One of the primary means of preventing seizures is to get enough sleep. Another good preventative effort is for patients and family members to receive annual flu shots. When patients do get sick, proper hydration, fever treatment, and treatment of infection is essential. While they are not necessarily present at a higher rate in SWS patients, iron deficiency and anemia should be diagnosed and properly treated.

Presymptomatic treatment with low-dose aspirin has been offered to a small group of infants and young children prior to the onset of seizures or strokes; the range of outcomes by neuroscore a few years later is variable and the numbers likely too small to determine if there is an effect [21**]. Presymptomatic treatment with phenobarbital has been studied, and was associated with reduced risk for cognitive impairment in patients receiving phenobarbital before their first seizure, versus those receiving it after their first seizure [22]. We offer anticonvulsants (leviteracetam) and low-dose aspirin to presymptomatically diagnosed infants with extensive bilateral brain involvement, whose entire brains are at risk for injury and atrophy since their prognosis is poor. Very few infants have been diagnosed presymptomatically and treated at this point, and more experience is needed to comment on effectiveness of this approach (unpublished data).

A subset of patients with SWS fail medical management and have frequent and/or prolonged seizures despite two or more anticonvulsants and low-dose aspirin. These patients should be considered for surgery. The decision to proceed with surgery is made easier if the patient already has hemiparesis and a visual field deficit, as is often the case when their seizures are frequent, or if they are a candidate for a focal resection that will not be expected to leave them with a motor deficit. Please see below for more information on surgical management of seizures in Sturge-Weber syndrome.

Modified Atkins Diet and Ketogenic diet-Medications are effective in preventing seizures in roughly $50 \%$ of patients according to the literature [23, 24]; with the use of newer anticonvulsants and low-dose aspirin this percentage is probably higher. For the remaining patients, surgery and lifestyle changes may become options. The Modified Atkins Diet (MAD) may be useful in controlling seizure activity. Unlike the ketogenic diet, which begins with calorie and fluid restriction, MAD does not begin this way. This modification may help to avoid triggering stroke-like episodes, and because MAD is less restrictive than the full ketogenic diet, it may increase adherence in young patients. In a study of five patients on the MAD, $60 \%$ saw a greater than $50 \%$ reduction in seizure activity after three months, and the remaining $40 \%$ saw a $25 \%$ percent reduction in seizure frequency; one of 
these patients gained complete control at 6 months and one had $90 \%$ control at 6 months [25]. However, none of the five subjects have remained on the diet (unpublished data). The ketogenic diet has also been safely used as well (unpublished data) and can be helpful, especially in infants.

\section{Hemispherectomy}

Standard procedure: There are several different techniques currently in use, including the functional hemispherectomy, anatomic hemipherectomy and hemispherotomy [26]. In these procedures, regions of the brain are surgically removed, or connections are severed in an attempt to control seizure activity. Recently a few surgical series have been published [27, $28,29]$.

Contraindications: Since the goal is to remove epileptic, diseased brain tissue, patients with bilateral brain involvement typically are not good surgical candidates. However, hemispherectomy or focal resections have been done rarely even in these cases when a child is very severely affected by seizures, has failed extensive medical attempts to manage their seizures and seizure monitoring suggests that most of their seizures are coming from one side of the brain or one region [30].

Complications: Motor deficits and visual field cut (this is minimized if the patient already has these deficits prior to surgery), infection, intracranial hemorrhage, hydrocephalus/ ventriculoperitoneal shunt placement, and rare mortality.

Special points: Today's procedures have drastically improved and offer a much better option for reduced complications and side-effects. SWS has been noted as a condition that responds exceptionally well to hemispherectomy for seizure control [26]. Due to increased plasticity and ability for remodeling in young brains, hemispherectomies completed earlier in life may be better at reducing functional deficits [31]. However, there is not a consensus that earlier hemispherectomies are always better. In the case of SWS, later hemispherectomies have been linked to positive outcome [27]. Patients who have daily or weekly seizures despite aggressive medication, and already present secondary symptoms such as hemiparesis, and visual field deficits are generally considered the best candidates for hemispherectomy [9]. Those with bilateral brain involvement can potentially be considered for this procedure if the seizures originate in one hemisphere only.

Cost / cost effectiveness: As with any surgery, this procedure is costly. It involves an inpatient stay in the hospital, anesthesia, pre- and postoperative monitoring, surgical rehabilitation, and recovery, in addition to other costs.

\section{Focal Brain Resection}

Standard procedure: Removing the region of the brain where seizures originate

Contraindications: Patients with seizures originating in different regions of the brain, or patients with seizures originating in an eloquent brain region are poor candidates for this procedure.

Curr Treat Options Neurol. Author manuscript; available in PMC 2015 July 01. 
Complications: Infection, intracranial hemorrhage, mortality

Special points: This surgical procedure has more limited postoperative morbidity, and should be considered before hemispherectomy in patients with localized seizures. It is also much better at preventing visual field cuts, and hemiparesis that are highly associated with hemispherectomy. It is a good option for patients with frequent seizures, which have not responded to at least two different seizure medications and low-dose aspirin [32]. In patients with SWS however, it has been shown that risk of seizure recurrence is higher with focal resections than with hemispherectomy [28, 29].

Cost / cost effectiveness: As with any surgery, this procedure is costly. It involves an inpatient stay in the hospital, anesthesia, pre- and postoperative monitoring, surgical rehabilitation, and recovery, in addition to other costs.

\section{Stroke-Like Episodes}

SWS severity has been associated with thrombosis and subsequent venous stasis, which can both be improved through the use of antiplatelet medications [21**]. While it's use remains controversial and there has never been a randomized, placebo controlled trial, low-dose aspirin (3-5 mg/kg/day) has been shown to be safe for patients with SWS, and to be helpful in reducing stroke-like episodes and seizures [21**, 33, 34]. By preventing these episodes, we hope to prevent cognitive problems, and functional disability. Since children most commonly have their deteriorations when ill, and low-dose aspirin has never been associated with Reye syndrome even in the thousands of children world-wide that use it for secondary stroke reduction, it is not recommended that the dose be reduced when ill. The flu shot is recommended annually to keep the patient healthy. In our center, a low dose aspirin regimen is often initiated before seizure activity begins, in patients with risk factors such as early handedness or visual gaze preference. It can also be initiated after imaging confirms brain involvement, even if no symptoms have developed. Aspirin therapy can be initiated in patients as young as 1 month of age, if seizures or the above risk factors are present.

Low Dose Aspirin [21**]

Standard dosage-3-5 mg/kg/day

Contraindications-Those having experienced negative reactions to aspirin in the past, aspirin allergy.

Main drug interactions-Aspirin is safe to use with all of the commonly prescribed anticonvulsants

Main side effects-Increased bruising and nosebleeds

Special points-Aspirin is recommended to help prevent venous stasis and microvascular thrombosis, which can both contribute to neurological decline. Previous studies have shown aspirin to be safe in the SWS population, as well as potentially useful in reducing seizure activity and stroke-like episodes. Rare sub-dural or sub-galeal bleeds have been reported 
with minor trauma however such bleeds in SWS have also been reported without associated low-dose aspirin use thus cause/effect relationship has not been established.

Cost / cost effectiveness-Inexpensive

\section{Headaches}

For patients with SWS, headaches and migraines can be even more disruptive and difficult to treat than seizures [35]. The relationship between migraines and seizures is complex, with headaches often triggering seizures, and seizures frequently triggering headaches. Migraines can first be treated with standard, over the counter painkillers. When they occur frequently or with greater severity, preventative medications can be considered. In a survey of 74 patients with SWS and migraines, 35\% utilized preventative migraine medication [36]. Ideally, preventative medications can be used that help to manage seizures and headaches concurrently, such as topiramate, valproate, and gabapentin. These medications have not been specifically studied for the treatment of seizures and/or migraines in SWS, however. [9]. The same survey of 74 patients also found that $22 \%$ of patients had tried triptans, many with improvements and no complications [36]. It is important not only to address headaches to improve quality of life, but also to try and prevent triggering additional seizures or strokelike episodes.

\section{Ophthalmologic Symptoms}

For glaucoma, medication is generally the first line of attack, although it is often not enough. The best studied topical medication is latanoprost, with research showing effective control of intraocular pressure in up to $50 \%$ of patients $[37,38,39]$. When medication alone is unable to reduce elevated eye pressure, it is often necessary to resort to surgery to maintain optimal eye function. Common surgical therapies include trabeculectomy, trabeculotomy and goniotomy in infants and children, as well as valve drainage implants, nonpenetrating sclerectomy and ciliodestructive procedures in adults[40, 41]. The most successful treatment is often a combination of two of the above surgical techniques [37]. The goal of both eye drops and surgery is to reduce fluid in the eye so as to reduce eye pressure and reduce the risk if ophthalmic nerve infarction and vision loss in that eye [37]. Even with the best treatment, infants with florid glaucoma may not respond to treatment and bupthalmos (eye enlargement) and amblyopia may result [42].

\section{Dermatologic Symptoms}

For the skin involvement, laser treatment can result in lightening of the port-wine birthmark. Laser treatment is also thought to reduce the long-term risk of soft and bony tissue hypertrophy and the attendant functional impairments related to swallowing, speaking, breathing, vision, and hearing [43]. The current method for lightening port-wine birthmarks is the flashlamp-pumped PDL, which targets the port-wine vasculature without affecting the surrounding epidermis or dermis [44]. While PDL can significantly lighten the majority of port-wine birthmarks, it is most effective when the birthmark is pink and flat, and thus when begun in infancy, and frequently requires maintenance treatments on an ongoing basis [45, 46]. Some port-wine birthmarks are "PDL resistant" and do not lighten with this current therapy [47]. For others, progressive lightening ends after six to ten treatments, so newer and 
more effective lasers are being researched to meet these needs [44]. Research is also underway to evaluate the combination of laser treatment with an mTOR inhibitor or other anti-angiogenesis agents and in the future this approach may provide more effective management [48*].

\section{Endocrine Function}

Endocrine issues are more common in patient with SWS than in the general population. They have an increased risk of growth hormone deficiency, which presents in childhood with growth below the $5^{\text {th }}$ percentile or significantly below the mean parental height [49]. Growth hormone deficiency may be screened for in children over the age of 2, where this diagnosis is suspected, with a serum IGF-1 level. However formal growth hormone stimulation testing should be done to confirm the diagnosis. Patients who are deficient may be treated, however, in our experience it is not uncommon for their treatment to be complicated by a worsening of their seizure status (unpublished data). Central hypothyroidism is also seen and probably relates to the use of anticonvulsants [50]. As we reported, testing by free $\mathrm{T} 4$ by dialysis is required to make the accurate diagnosis of central hypothyroidism [51]. Patients with verifiably and consistently low levels of free thyroxine, and with normal morning cortisol levels, should be started on levothyroxine. Patients without normal cortisol levels can experience adrenal insufficiency due to increased clearance of cortisol by thyroxine replacement. For patients with inconsistently low levels of free thyroxine, levothyroxine should not be initiated, but careful monitoring is important [50]. In our experience, treatment of children with true central hypothyroidism is anecdotally helpful to their stabilization and progress.

\section{Cognitive/Psychological Function and Hemiparesis}

Cognitive impairments may range from attention problems, to mild learning disability, to severe cognitive impairment. Attention problems, mood issues and various cognitive difficulties, depending on the side, region of brain involvement and severity of seizures may contribute significantly to quality of life and academic performance [15**]. Early seizure onset and bilateral brain involvement are both risk factors for cognitive impairment. In addition, patients with hemiparesis are more likely to have general cognitive impairments [52]. For patients displaying these risk factors, neuropsychological testing performed between ages 3 and 4 can help to identify any specific deficits or concerns [9]. Those patients with greater cognitive impairment are shown to also have a greater rate of emotional and behavioral issues [53,54]. Patients with cognitive deficits and attention problems can benefit from specialized education services, behavioral psychology intervention, and stimulant use. Stimulants have been shown to positively impact attention problems in SWS patients [55]. To address hemiparesis, physical and occupational therapy can be incorporated at an early age to improve motion and fine motor skills; some work with constraint therapy has been done with these patients and has been shown to be helpful [56].

\section{Acknowledgments}

The authors gratefully acknowledge funding from Hunter's Dream for a Cure Foundation and from The Brain Vascular Malformation Consortium (BVMC; U54NS065705), which is a part of the National Institutes of Health (NIH) Rare Disease Clinical Research Network (RDCRN), supported through collaboration between the NIH 
Office of Rare Diseases Research (ORDR) at the National Center for Advancing Translational Science (NCATS), and the National Institute of Neurological Disorders and Stroke (NINDS). The content is solely the responsibility of the authors and does not necessarily represent the official views of the National Institutes of Health.

\section{References and Recommended Reading}

Papers of particular interest, published recently, have been highlighted as

* Of importance

** Of major importance

1. Melancon JM, Dohil MA, Eichenfield LF. Facial port-wine stain: When to worry? Pediatr Dermatol. 2012; 29(1):131-3. [PubMed: 22256997]

2. Piram M, Lorette G, Sirinelli D, et al. Sturge-Weber syndrome in patients with facial port-wine stain. Pediatr Dermatol. 2012; 29(1):32-7. [PubMed: 21906147]

3. Enjolras O, Riche MC, Merland JJ. Facial port-wine stains and Sturge-Weber syndrome. Pediatrics. 1985; 76:48-51. [PubMed: 4011357]

4. Tallman B, Tan OT, Morelli JG, et al. Location of port-wine stains and the likelihood of ophthalmic and/or central nervous system complications. Pediatrics. 1991; 87:323-7. [PubMed: 1805804]

5*. Nabbout R, Juhasz C. Sturge-Weber syndrome. Hand Clin Neurol. 2013; 111:315-21. This article addresses the most updated techniques for MRI imagining in Sturge-Weber syndrome. It is important to understand these methods in order to accurately diagnose brain involvement and to prescribe treatment options accordingly.

6. Ewen JB, Kossoff EH, Crone NE, et al. Use of quantitative EEG in infants with port-wine birthmark to assess for Sturge-Weber brain involvement. Clin Neurophysiol. 2009; 120(8):1433-40. [PubMed: 19589723]

7. Hatfield LA, Crone NE, Kossoff EH, et al. Quantitative EEG asymmetry correlates with clinical severity in unilateral Sturge-Weber syndrome. Epilepsia. 2007; 48(1):191-5. [PubMed: 17241228]

$8^{* *}$. Shirley MD, Tang H, Gallione CJ, et al. Sturge-Weber syndrome and port-wine stains caused by somatic mutation in GNAQ. N Engl J Med. 2013; 368(21):1971-9. This article uncovers the somatic mosaic genetic basis for port wine birthmarks and Sturge-Weber syndrome. The article also utilizes transient transfections to determine the impact of the GNAQ mutation upon downstream phosphorylated proteins. [PubMed: 23656586]

9. Comi AC. Presentation, diagnosis, pathophysiology, and treatment of the neurological features of Sturge-Weber syndrome. Neurologist. 2011; 17(4):179-184. [PubMed: 21712663]

10. Traub R, Riley C, Horvath S. Teaching NeuroImages: Sturge-Weber syndrome presenting in a 58year old woman with seizures. Neurology. 2010; 75(12):e52. [PubMed: 20855845]

11. Kumar KR, Hon K, Schultz D, et al. Transient changes on brain magnetic resonance imaging in a patient with sturge-weber syndrome presenting with hemiparesis. Neurologist. 2009; 15(6):351-4. [PubMed: 19901718]

12. Zolkipli Z, Aylett S, Rankin PM, et al. Transient exacerbation of hemiplegia following minor head trauma in Sturge-Weber syndrome. Dev Med Child Neurol. 2007; 49:697-699. [PubMed: 17718827]

13. Bebin EM, Gomez MR. Prognosis in Sturge-Weber disease: comparison of unihemispheric and bihemispheric involvement. J Child Neurol. 1988; 3:181-184. [PubMed: 3209844]

14. Oakes WJ. The natural history of patients with the Sturge-Weber syndrome. Pediatr Neurosurg. 1992; 18:287-90. [PubMed: 1476938]

$15^{* *}$. Raches D, Hiscock M, Chapieski L. Behavioral and academic problems in children with SturgeWeber syndrome: differences between children with and without seizures. Epilepsy Behav. 2012; 25(3):457-63. This article addresses the neurological cognitive impairments that can accompany Sturge-Weber syndrome. As cognitive impairments are thought to be a direct result of seizure activity, successful treatment is necessary to prevent them. [PubMed: 23000106]

16. Namer IJ, Battaglia F, Hirsch E, et al. Subtraction ictal SPECT co-registered to MRI (SISCOM) in Sturge-Weber syndrome. Clin Nucl Med. 2005; 30:39-4. [PubMed: 15604970] 
17. Aylett SE, Neville BG, Cross JH, et al. Sturge-Weber syndrome: cerebral haemodynamics during seizure activity. Dev Med Child Neurol. 1999; 41:480-485. [PubMed: 10454232]

18. Juhasz C, Lai C, Behen ME, et al. White matter volume as a major predictor of cognitive function in Sturge-Weber syndrome. Arch Neurol. 2007; 64(8):1169-74. [PubMed: 17698708]

19. Kossoff EH, Hatfield LA, Ball KL, et al. Comorbidity of epilepsy and headache in patients with Sturge-Weber syndrome. J Child Neurol. 2005; 20(8):678-82. [PubMed: 16225815]

20. Vainionpaa LK, Mikkonen K, Rattya J, et al. Thyroid function in girls with epilepsy with carbamazepine, oxcarbazepine and valproate. Pediatr Int. 2004; 45:197-203.

$21^{* *}$. Lance EI, Sreenivasan AK, Zabel TA, et al. Aspirin use in Sturge-Weber syndrome: side effects and clinical outcomes. J Child Neurol. 2012; 28(2):213-8. This article offers direct evidence for the safety of aspirin use among Sturge-Weber syndrome patients and suggests that aspirin is a practical and useful treatment for these patients. [PubMed: 23112247]

22. Ville D, Enjolras O, Chiron C, et al. Prophylactic antiepileptic treatment in Sturge-Weber disease. Seizure. 2002; 11(3):145-50. [PubMed: 12018956]

23. Arzimanoglou A, Aicardi J. The epilepsy of Sturge-Weber syndrome: clinical features and treatment in 23 patients. Acta Neurol Scand Suppl. 1992; 140:18-22. [PubMed: 1279926]

24. Sujansky E, Conradi S. Sturge-Weber syndrome: Age of onset of seizures and glaucoma and the prognosis for affected children. J Child Neurol. 1995; 10:49-58. [PubMed: 7769179]

25. Kossoff EH, Borsage JL, Comi AM. A pilot study of the modified Atkins diet for Sturge-Weber Syndrome. Epilepsy Res. 2010; 92(2-3):240-3. [PubMed: 20934305]

26. Schramm J, Kuczaty S, Sassen R, et al. Pediatric functional hemipherectomy: outcome in 92 patients. Acta Neurochir. 2012; 154(11):2017-28. [PubMed: 22941395]

27. Kossoff EH, Buck C, Freeman JM. Outcomes of 32 hemispherectomies for Sturge-Weber syndrome worldwide. Neurology. 2002; 59(11):1735-8. [PubMed: 12473761]

28. Bourgeois M, Crimmins DW, de Oliveira RS, et al. Surgical treatment of epilepsy in Sturge-Weber syndrome in children. J Neurosurg. 2007; 106(1 Suppl):20-8. [PubMed: 17233308]

29. Arzimanoglou AA, Andermann F, Aicardi J, et al. Sturge-Weber syndrome: indications and results of surgery in 20 patients. Neurology. 2000; 55:1472-9. [PubMed: 11094100]

30. Tuxhorn IE, Pannek HW. Epilepsy surgery in bilateral Sturge-Weber syndrome. Pediatr Neurol. 2002; 26(5):394-7. [PubMed: 12057803]

31. Wiebe S, Berg AT. Big epilepsy surgery for little people: what's the full story on hemispherectomy? Neurology. 2013; 80(3):232-3. [PubMed: 23223536]

32. Lo W, Marchuck DA, Ball KL, et al. Updates and future horizons on the understanding, diagnosis, and treatment of Sturge-Weber syndrome brain involvement. Dev Med Child Neurol. 2012; 54:214-223. [PubMed: 22191476]

33. Maria BL, Neufeld JA, Rosainz LC, et al. Central nervous system structure and function in SturgeWeber syndrome: evidence of neurologic and radiologic progression. J Child Neurol. 1998; 13(12):606-18. [PubMed: 9881531]

34. Udani V, Pujar S, Munot $P$, et al. Natural history and magnetic resonance imagine follow-up in 9 Sturge-Weber syndrome patients and clinical correlation. J Child Neurol. 2007; 22(4):479-83. [PubMed: 17621534]

35. Iizuka T, Sakai F, Yamakawa K, et al. Vasogenic leakage and the mechanism of migraine with prolonged aura in Sturge-Weber syndrome. Cephalalgia. 2004; 24:767-770. [PubMed: 15315534]

36. Kossoff EH, Balasta M, Hatfield LM, et al. Self-reported treatment patterns in patients with Sturge-Weber syndrome and migraines. J Child Neurol. 2007; 22:720-726. [PubMed: 17641257]

37. Basler L, Sowka J. Sturge-Weber syndrome and glaucoma. Optometry. 2011; 82(5):306-9. [PubMed: 21524602]

38. Ong T, Chia A, Nischal KK. Latanoprost in port-wine stain related pediatric glaucoma. $\mathrm{Br} \mathbf{J}$ Ophthamol. 2003; 87:1091-3.

39. Yang CB, Freedman SF, Myers JS, et al. Use of latanoprost in the treatment of glaucoma associated with Sturge-Weber syndrome. Am J Ophthamol. 1998; 126:600-2.

40. Patrianakos TD, Nagao K, Walton DS. Surgical management of glaucoma with the Sturge-Weber syndrome. Int Ophthamol Clin. 2008; 48:63-78.

Curr Treat Options Neurol. Author manuscript; available in PMC 2015 July 01. 
41. Audren F, Abitbol O, Dureau P, et al. Non-penetrating deep sclerectomy for glaucoma associated with Sturge-Weber syndrome. Acta Ophthamol Scand. 2006; 84:656-60.

42. Saltzmann RM, Reinecke S, Lin X, et al. Long-term outcomes of a pseudo 360-degree trabeculotomy ab externo technique for congenital glaucoma at children's medical center. Clin Ophthamol. 2012; 6:289-98.

43. Eivazi B, Roessler M, Pfützner W, et al. Port-wine stains are more than skin deep! Expanding the spectrum of extracutaneous manifestations of nevi flammei of the head and neck. Eur J Dermatol. 2012; 22(2):246-51. [PubMed: 22285557]

44. Garzon MC, Huang JT, Enjolras O, et al. Vascular malformations: Part I. J Am Acad Dermatol. 2007; 56(3):371-4.

45. Orten SS, Waner M, Flock S, et al. Port-wine stains. An assessment of 5 years of treatment Arch Otolaryngol-Head Neck Surg. 1996; 122:1174-1179.

46. Lam SM, Williams EF 3rd. Practical considerations in the treatment of capillary vascular malformations, or port wine stains. Facial Plast Surg. 2004; 20(1):71-6. [PubMed: 15034817]

47. Kane KS, Smoller BR, Fitzpatrick RE, et al. Pulsed-dye resistant port-wine stains. Arch Dermatol. 1996; 132:839-841. [PubMed: 8678586]

48*. Tan W, Jia W, Sun V, et al. Topical rapamycin suppresses the angiogenesis pathways induced by pulsed dye laser: molecular mechanisms of inhibition of regeneration and revascularization of photocoagulated cutaneous blood vessels. Lasers Surg Med. 2012; 44(10):796-804. This article advances a new approach in the treatment of port-wine birthmark which combines laser treatments with anti-angiogenesis treatment to prevent the regeneration of blood vessels obliterated by the laser treatment. [PubMed: 23213008]

49. Miller RS, Ball KL, Comi AM, et al. Growth hormone deficiency in Sturge-Weber syndrome. Arch Dis Child. 2006; 91(4):340-1. [PubMed: 16551788]

50. Comi AM, Bellamkonda S, Ferenc LM, et al. Central hypothyroidism and Sturge-Weber syndrome. Pediatr Neurol. 2008; 39(1):58-62. [PubMed: 18555176]

51. Saddique L, Sreenivasan A, Comi AM, et al. Important of utilizing a sensitive free thyroxine assay in Sturge-Weber syndrome. J Child Neurol. 2013; 28(2):269-74. [PubMed: 23112245]

52. Reesman J, Gray R, Suskauer SJ, et al. Hemiparesis is a clinical correlate of general adaptive dysfunction in children and adolescents with Sturge-Weber syndrome. J Child Neurol. 2009; 24:701-708. [PubMed: 19359254]

53. Chapieski L, Friedman D, Lachar D. Psychological functioning in children and adolescents with Sturge-Weber syndrome. J Child Neurol. 2000; 15(10):660-5. [PubMed: 11063079]

54. Sujansky E, Conradi S. Outcome of Sturge-Weber syndrome in 52 adults. Am J Med Genet. 1995; 57(1):35-45. [PubMed: 7645596]

55. Lance, EI.; Lanier, KE.; Zabel, TA.; Comi, AC. Stimulant Use in Patients with Sturge-Weber Syndrome: Safety and Efficacy. Abstract presented at Child Neurology Society; Nov. 2012;

56. Suskauer SJ, Trovato MK, Zabel TA, et al. Physiatric findings in individuals with Sturge-Weber syndrome. Am J Phys Med Rehabil. 2010; 89(4):323-30. [PubMed: 20068437]

Curr Treat Options Neurol. Author manuscript; available in PMC 2015 July 01. 


\section{Opinion Statement}

We try to see the babies prior to the onset of symptoms so that their parents can receive anticipatory guidance regarding seizures and how to recognize and respond to them and so that proper referrals to ophthalmology can be made. If there is any concern on history, exam or EEG then we obtain an MRI with contrast. If presymptomatic diagnosis of brain involvement is made then treatment with low-dose aspirin is offered and if the brain involvement is extensively bilateral then an anticonvulsant such as levetiracetam is offered as well. Seizures are treated aggressively with goal of obtaining and maintaining complete seizure suppression as much as possible often with a combination of low-dose aspirin and two anticonvulsants such as levetiracetam and oxcarbazepine. For many patients, this will provide adequate control of their seizures and stroke-like episodes. If the patient fails medical management and seizures are regular and accompanied by plateaued development, significant hemiparesis and visual field deficit and the patient is unilaterally involved and a surgical candidate then surgical management is urged. When the seizures are less regular, little or no hemiparesis or visual field deficit exist, and development is reasonable then this decision is more difficult. For bilaterally involved patients surgery is usually not a good option unless seizures are very severe and mostly coming from one side. Other therapeutic options include the ketogenic/Atkins diet and vagal nerve stimulator although in our experience these usually do not result in cessation of seizures. Endocrine problems occur with increased frequency and must be treated when they are present. The recent discovery of the somatic mutation causing SturgeWeber syndrome holds promise for new treatment options in the future.

Curr Treat Options Neurol. Author manuscript; available in PMC 2015 July 01. 